\title{
Journée scientifique de la Société de pathologie exotique, Peau et maladies tropicales 21 novembre 2019, École du Val-de-Grâce, Paris
}

\section{Scientific Day of the Society of Exotic Pathology: Skin and Tropical Diseases November 21, 2019, Val-de-Grâce School, Paris}

\author{
P. Gazin · P. Marty $\cdot$ E. Pichard $\cdot$ J. Chandenier $\cdot$ J. Jannin $\cdot$ Y. Buisson
}

Reçu le 15 novembre 2019; accepté le 19 novembre 2019

(C) Société de pathologie exotique et Lavoisier SAS 2019
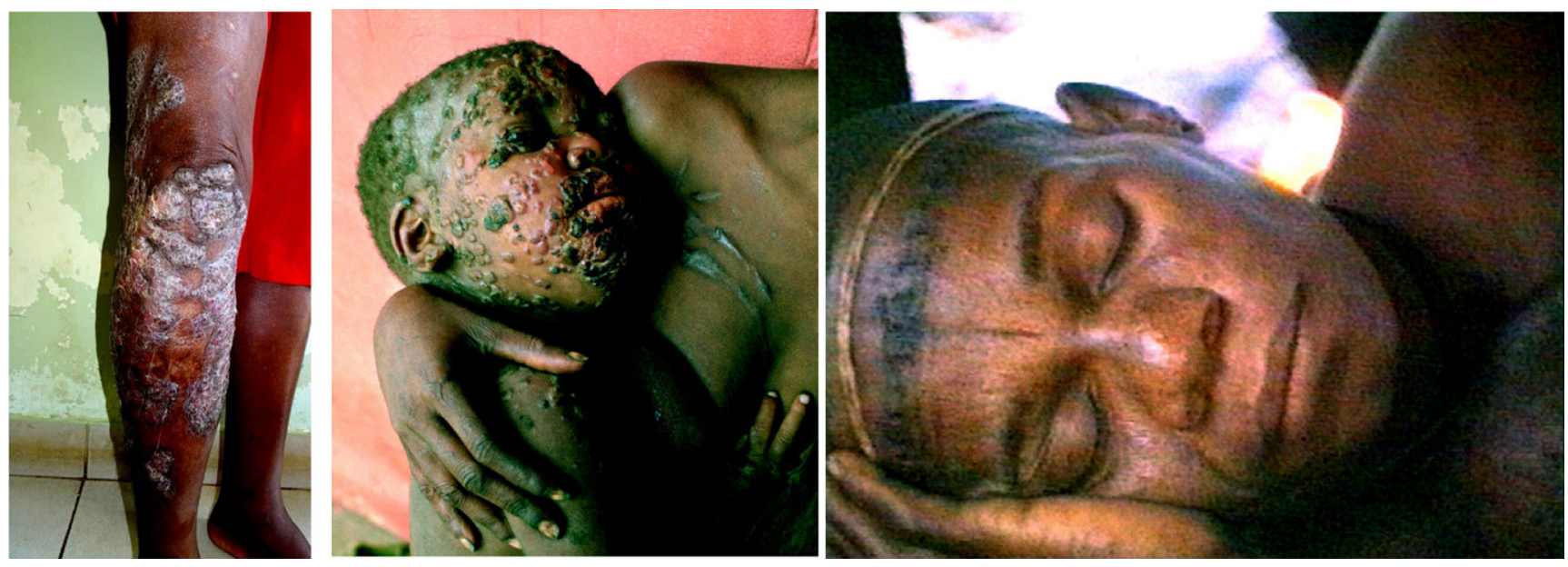

Fig. 1 Crédits photos : Chromoblastomycose (J. Chandenier) ; Kai Pian (A. Epelboin) ; Scarification en traitement de la migraine (A. Epelboin) / Chromoblastomycosis (J. Chandenier); Yaws Kai (A. Epelboin); Scarification used to treat migraine (A. Epelboin)

\section{Introduction}

La peau occupe une place particulière en médecine tropicale. D'une part, les infections bactériennes, parasitaires et fongiques sont fréquentes en milieu tropical, d'autre part, bon nombre de maladies systémiques ont une expression cutanée qui permet une orientation diagnostique clinique utile. Cela est aussi bien le cas en médecine du voyage et des migrants qu'à la portée des personnels de santé démunis de plateau technique sur le terrain. L'examen biologique de la peau, tissu accessible, permet des diagnostics peu coû- teux, en particulier au cours des maladies tropicales négligées comme la leishmaniose, la lèpre, la gale, les mycoses sous-cutanées, ou même plus récemment la trypanosomose africaine. Les lésions cutanées ont aussi une bonne valeur d'orientation au cours de maladies vectorielles, d'inoculation ou traumatiques. Enfin, objet d'admiration ou de stigmatisation, l'aspect de la peau est une préoccupation importante des populations tropicales menant parfois à des pratiques cosmétiques pathogènes.

Ces divers aspects seront abordés lors des différentes conférences de la Journée.

P. Gazin $(\bowtie) \cdot$ P. Marty $\cdot$ E. Pichard $\cdot$ J. Chandenier $\cdot$ J. Jannin

Y. Buisson

Comité scientifique, Société de pathologie exotique,

hôpital de la Pitié-Salpêtrière, pavillon Laveran,

47/83, bld de l'Hôpital, F-75013 Paris, France

e-mail : bulletin@pathexo.fr 


\section{Exposé d'ouverture}

\section{Anthropologie de la peau : esthétiques, soins et cliniques transculturelles en Afrique subsaharienne}

A. Epelboin

Médecin anthropologue, « retraité actif», CNRS-MNHNmusée de l'Homme, Paris, France

epelboinalain@gmail.com

Des chantres de la négritude, exaltant la beauté de la peau « noire » aux blanchiments volontaires, aux trafics criminels de fragments de corps d'albinos, la couleur de la peau est un critère de classification et de distinction sociales. Dans de nombreuses sociétés africaines, les peaux foncées sont dévalorisées au profit des peaux claires : le désir de reconnaissance sociale, l'idéologie esthétique dominante de la mondialisation conduit certain(e)s à employer des produits « dépigmentants » très dangereux pour leur santé et/ou leur esthétique future.

Les toilettes quotidiennes en Afrique subsaharienne (cf. vidéothèque SMM Canal U : http://www.canal-u.tv, mot clé : « toilette ») sont remarquables dès la naissance par leur tonus, le recouvrement intégral de l'enveloppe cutanée, l'usage « énergique » d'un outil abrasif (éponge végétale, fibre de palmier, chiffon, gant de toilette, morceau de filet de pêche, etc.), suivies - plus rarement précédées par l'application énergique d'un corps gras : il s'agit de fait de massages vigoureux, associés à une gymnastique précise, exécutés selon un schéma corporel culturellement original et transmis par les femmes, de génération en génération.

Chaque peau a sa propre odeur qui dépend des savons, des cosmétiques gras (beurre de karité plus ou moins raffiné, huile de palme ou de palmiste, beurre de cacao et produits industriels) et des parfums (faux vétiver = Andropogon gayanus, encens divers, parfums industriels), appliqués selon des schémas corporels et rituels propres à chaque culture. Cette odeur de l'enveloppe cutanée est aussi dépendante des aliments et condiments consommés, dont les principes odorants imprègnent les fluides corporels, comme le lait maternel, la sueur et les excreta. Au-delà de la couleur, l'odeur de la peau suscite des discriminations négatives, renvoyant à des déficits supposés d'hygiène, à l'animalité, à la sauvagerie pour les uns, à des odeurs d'urine, d'acide de batterie, de cadavre, pour les autres.

Certaines peaux, dont de nombreuses peaux africaines, font facilement des cicatrices chéloïdes très visibles ; elles peuvent être le résultat involontaire d'accidents, mais aussi de scarifications esthétiques - voire érogènes - ou thérapeutiques volontaires (extraction de pus, de sang, de « venin » et par illusionnisme d'objets « magiques ») ou administration de substances thérapeutiques curatives ou préventives (broyats de matières animales et végétales calcinées, mêlées à de l'huile de palme et à du sel végétal par exemple). Les cicatrices visibles de l'enveloppe cutanée sont alors des outils d'identifica- tion culturelle, d'affichage de statut social, des cartes d'identité, des mémoires d'histoires de vie, d'événements vécus, heureux, malheureux et morbides, des dossiers médicaux.

Les affections dermatologiques congénitales (ichtyose, vitiligo, etc.) ou acquises (abcès cutanés, ulcères phagédéniques, mycoses, zonas, onchocercose, lèpre, pian, etc.), de même que les maladies et malheurs en général relèvent de sémiologies et nosologies distinctes de celles de la biomédecine. Là où les explications épidémiologiques, statistiques et génétiques s'arrêtent, les ruptures d'interdits, alimentaires ou non, la mise en cause de sorciers dévoreurs, d'ancêtres, de génies, de malfaisants humains et non humains donnent du sens au mal, avec une priorité donnée au traitement de ces étiologies avant la cure générale ou locale proprement dite.

En médecine transculturelle, la cure d'affections cutanées chroniques passe par une prise en considération des représentations, usages et savoir-faire du patient, avec une attention toute particulière portée à la prévention des autoattouchements générateurs de réinfections et chronicisations.

\section{Session 1}

\section{Monkeypox et autres poxviroses}

\section{J.-J. Morand}

Service de dermatologie, 2 bld Sainte-Anne, 83000 Toulon, France

morandjj@aol.com

Les infections à poxvirus (PXV) doivent être bien connues du praticien exerçant notamment dans les pays à ressources faibles, car il s'agit d'agents viraux à haut risque épidémique : la variole (VARV), éradiquée, fait toujours l'objet d'une surveillance du fait d'un risque potentiel de réintroduction accidentelle ou volontaire par le bioterrorisme ; le Monkeypox virus (MXV) ou le Cowpox virus (CXV) sont considérés comme émergents avec un fort risque de diffusion du fait de l'augmentation des transports internationaux, de la mode des nouveaux animaux de compagnie, volontiers exotiques, et de la perte de la protection vaccinale antivariolique.

Le Molluscum contagiosum virus (MCV) s'en distingue, car il est à l'origine d'infections bénignes, spécifiquement cutanées. Particulièrement fréquent chez l'enfant, il constitue chez l'adulte un marqueur de risque d'infection sexuellement transmissible et peut révéler, en cas de profusion, le sida. La variole et le MCV ont un réservoir naturel humain; les autres PXV ont un réservoir naturel animal. Ces virus, dont le nom « pox » signifie pustule, ont un tropisme pour le tégument.

Le MXV mérite une description particulière car, rapporté seulement en 1970 chez un enfant dans l'ex-Zaïre (désormais République démocratique du Congo, RDC), dès les années 1980, il entraîne des épidémies en RDC touchant surtout les jeunes garçons en forêt qui se contaminent au 
contact des singes qu'ils chassent et qui n'avaient pas bénéficié de la protection vaccinale antivariolique. La maladie essaimait ensuite au Liberia, en Côte-d'Ivoire, au Sierra Leone, au Nigeria, au Bénin, au Cameroun, au Gabon et au Sud Soudan avec l'apparition d'une transmission interhumaine et d'une nouvelle souche virale. Or, en 2003, une mini-épidémie paucisymptomatique concernant une dizaine d'enfants aux États-Unis était causée par la transmission accidentelle de MXV par des rongeurs (rat de Gambie) importés illégalement du Ghana à des chiens de prairie dans une animalerie américaine. Ce virus est depuis sous surveillance, car il pourrait constituer une arme biologique ou devenir la « variole » du $\mathrm{XXI}^{\mathrm{e}}$ siècle...

\section{Session 2}

\section{Persistance de la lèpre et nouveaux challenges}

\section{E. Cambau}

Université de Paris, Inserm UR1137 IAME ; Centre national de référence des mycobactéries et de la résistance des mycobactéries aux antituberculeux (CNR-MyRMA), AP-HP, hôpital Lariboisière, Paris, France

emmanuelle.cambau@aphp.fr

La lèpre fait aujourd'hui partie des maladies tropicales, alors qu'elle a était présente sur tous les continents et latitudes jusqu'au $\mathrm{XX}^{\mathrm{e}}$ siècle. C'est d'abord l'amélioration des conditions de vie, de l'état nutritionnel et la diminution de la promiscuité qui ont permis la disparition des cas en Europe et autres pays de l'hémisphère nord, car la transmission interhumaine nécessite un contact étroit et prolongé, et l'installation de la maladie se fait préférentiellement sur un terrain fragile. Ensuite, la découverte de l'action antilépreuse de divers antibactériens dès les années 1950 (sulfamides, rifampicine, quinolones, etc.) a permis de traiter, de prendre en charge et de guérir les malades atteints de lèpre, quelle que soit leur forme clinique, et d'arrêter l'isolement à vie des patients censés être contagieux. Pourtant, après une réduction phénoménale du nombre de cas mondiaux, la lèpre persiste dans le monde avec un peu plus de 200000 nouveaux cas rapportés à l'OMS chaque année. Le plus grand nombre de cas se trouve en Inde et au Brésil, et malgré des programmes nationaux et internationaux menés dans ces deux pays, la diminution des cas n'est pas évidente.

En effet, plusieurs problèmes non résolus empêchent l'élimination au niveau mondial, dont l'OMS rêve depuis que la polychimiothérapie standard (dapsone + rifampicine + clofazimine) est disponible gratuitement pour tous les cas (1982). D'abord, du fait de traitements mal conduits et de l'usage très répandu des antibiotiques pour les autres infections, des cas de lèpre à bacilles résistants à la dapsone, à la rifampicine ou aux quinolones ont été observés dans plusieurs pays du monde.
Ensuite, les connaissances sur la bactérie causale restent limitées malgré le séquençage du génome en 2001 et le développement des techniques moléculaires, car cette bactérie ne peut être cultivée in vitro. Cela ne permet pas d'avoir un test microbiologique simple pour faire le diagnostic de la maladie, en dehors de l'examen microscopique des frottis cutanés ou de la PCR qui sont positifs pour les formes les plus multibacillaires ou lépromateuses. Cela ne permet pas non plus de bien comprendre la réponse immunologique qui est complexe et qui a, non seulement, une grande importance dans la détermination de la forme clinique de lèpre et des atteintes cutanées et neurologiques qui vont survenir, mais aussi dans le développement de l'infection vers la maladie. Bien que cette réponse immunologique soit théoriquement proche de celles des autres maladies à mycobactéries dont la tuberculose, il n'existe pas de test biologique fiable pour faire le diagnostic de l'infection, c'est-à-dire un test équivalent à l'intradermoréaction ou les tests IGRA pour la tuberculose. Enfin, lorsque le patient est guéri microbiologiquement, la prise en charge des séquelles (neurologiques, cutanées, psychologiques entre autres) et des réactions immunologiques survenant pendant et après le traitement antibiotique est encore très insuffisante et n'est pas accompagnée (gratuité des soins et du traitement) ni standardisée. C'est pourquoi il est important de reprendre les recherches fondamentales, microbiologiques, immunologiques, cliniques et épidémiologiques si on veut espérer agir sur le contrôle de la lèpre. Il est aussi demandé aux pays endémiques d'augmenter le niveau de vie et le niveau sanitaire dans les régions concernées et aux entreprises industrielles d'aider à mettre à disposition des tests diagnostiques et de nouveaux traitements plus simples. Mieux diagnostiquer une maladie chez des populations qui étaient, jusque-là, sans accès à la santé, et voir une augmentation du nombre des cas détectés, pourrait cependant décourager les programmes de lutte. La discrimination et le stigma attaché à la lèpre sont encore présents dans de nombreux pays et cultures, et pourraient également empêcher certains pays de mener une action efficace.

\section{Dépigmentation cosmétique et ses risques}

\section{A. Mahé}

Service de dermatologie, hôpital Pasteur (HCC), Colmar, France

maheant@yahoo.fr

L'utilisation cosmétique de produits à visée dépigmentante est une pratique courante dans les populations dont les individus présentent une peau fortement pigmentée. La répartition géographique de cette pratique dépasse largement l'Afrique subsaharienne, continent où elle a été historiquement décrite ; dans certaines régions, une proportion importante de femmes peuvent être concernées ; une utilisation masculine est plus rarement rencontrée, mais n'est pas exceptionnelle. Les produits utilisés, souvent à base de principes actifs à usage 
normalement médical et détournés (corticoïdes locaux), ou même parfois carrément prohibés (dérivés mercuriels), sont employés quotidiennement, généralement sur de larges surfaces corporelles. Récemment, le glutathion s'est révélé en tant que dépigmentant émergeant. La pratique de dépigmentation cosmétique est susceptible d'être à l'origine de nombreuses complications dermatologiques, nombreuses et variées, mais également parfois de complications systémiques, moins connues et d'autant plus redoutables.

Au cours de notre présentation, un panorama de l'épidémiologie, des données pharmacologiques, des modalités pratiques et des complications dermatologiques et générales concernant la dépigmentation cosmétique sera présenté, ainsi qu'une tentative d'analyse des motivations ; enfin, des propositions à visée préventive seront effectuées.

\section{Session 3}

\section{Dermatoses tropicales et du voyageur}

\section{E. Caumes}

Service des maladies infectieuses et tropicales, groupe hospitalier Pitié-Salpêtrière, Paris Sorbonne Université, France

eric.caumes@aphp.fr

L'épidémiologie des dermatoses dites tropicales varie selon l'endroit de la planète. Dans les pays tropicaux, la situation dépend du milieu géographique. Les maladies infectieuses banales (gale, impétigo, teignes) prédominent en milieu rural. Les maladies dermatologiques cosmopolites (dermatite atopique, dermite de contact, toxidermies, urticaire, acné, verrues, etc.) deviennent de plus en plus fréquentes en milieu urbain. Dans les deux cas, et surtout en Afrique subsaharienne, viennent s'y ajouter les complications cutanées de la dépigmentation volontaire à visée cosmétique et le problème des mutilations génitales féminines. Dans les pays occidentaux, la situation varie en fonction du type de voyageur. Les dermatoses tropicales représentent moins de $20 \%$ de la pathologie dermatologique du voyageur ; et il s'agit principalement de Larva migrans cutanée ankylostomienne (LMCa), éventuellement de myiase furonculeuse et de leishmaniose cutanée localisée. Chez les migrants, les dermatoses se confondent progressivement avec celles du pays d'accueil. Chez les réfugiés, ce sont les dermatoses de la promiscuité (gale, impétigo) qui sont au premier plan. Dans tous les cas, les dermatoses emblématiques de la médecine tropicale (lèpre, tréponématoses non vénériennes, onchocercose, dracunculose, filarioses lymphatiques, loaose, etc.) sont de moins en moins observées, et certaines ont quasiment disparu.

Le voyageur est une sentinelle épidémiologique. Et les dermatoses observées dans ce contexte sont un bon reflet de celles qui existent dans les pays tropicaux visités. Il existe toutefois deux biais à prendre en compte. Le recrutement se fait dans des services de maladies infectieuses et tropicales, sous-estimant ainsi les dermatoses d'origine non infectieuse. Le type de voyageur est dominé par les touristes qui sont surexposés au risque de LMCa sur les plages tropicales. Mais les études nationales comme internationales montrent que les principales causes de consultation dermatologique au retour de voyages sont les conséquences des piqures d'insectes et d'arthropodes et les infections cutanées bactériennes banales (impétigo, ecthyma, folliculite, furoncle, dermohypodermite bactérienne aiguë). La forme clinique peut orienter vers l'agent pathogène, Streptococcus pyogenes (impétigo, ecthyma, dermohypodermite bactérienne aiguë) ou Staphylococcus aureus (impétigo, folliculite, furoncle). Le prélèvement microbiologique, quand il est possible, est indispensable non seulement pour établir un diagnostic plus difficile (mélioïdose, diphtérie, charbon, mycobactériose, trypanosomose africaine, etc.), mais aussi pour identifier l'agent microbien responsable, et en tester la sensibilité. Car s'il existe une touche tropicale dans ces infections cutanées banales et cosmopolites, elle concerne la résistance du staphylocoque doré aux antibiotiques, dont les contours varient selon la région visitée. Il a été montré environ un quart d'échec de l'antibiothérapie présomptive antistaphylococcique si l'on se fondait sur les recommandations nationales. Il vaudrait donc mieux se fonder sur celles en place dans le pays de séjour pour décider de l'antibiothérapie empirique antistaphylococcique.

Ainsi, les dermatoses tropicales sont de moins en moins tropicales quand l'on se réfère aux dermatoses historiques. Pour autant, les dermatoses tropicales existent encore ; mais leur diminution les rend de plus en plus difficiles à reconnâtre. Il faut donc rester le plus rigoureux possible dans la démarche diagnostique.

\section{Trypanosomose humaine africaine : réservoir cutané et diagnostic}

\section{B. Rotureau}

Groupe transmission des trypanosomes, unité de biologie cellulaire des trypanosomes, Inserm U1201, département des parasites et insectes vecteurs, Institut Pasteur, Paris, France brice.rotureau@pasteur.fr

L'OMS vise à éliminer la trypanosomose humaine africaine (THA) en tant que problème de santé publique en Afrique de l'Ouest d'ici 2020. Cet objectif a été rendu possible par le succès des mesures de surveillance des 25 dernières années ayant conduit à la déclaration de moins de 1500 nouveaux cas en 2017. Ces résultats reposent principalement sur la détection des trypanosomes dans le sang par un test sérologique suivi d'une confirmation parasitologique. Cependant, la densité parasitaire dans le sang fluctue périodiquement pendant une infection et peut parfois être très faible, voire indécelable. De plus, chez les personnes séropositives « asymptomatiques » 
ou « trypanotolérantes », les parasites sanguins restent indétectables, laissant en suspens la question du possible rôle de réservoir de ces infections latentes.

Dans ce contexte, nous avons récemment démontré en modèle murin que les trypanosomes se développent d'abord dans le compartiment extravasculaire, en particulier dans la peau, avant d'être détectés dans le sang. Contrairement aux parasites sanguins, les trypanosomes cutanés sont observés en permanence et avec une distribution dynamique au cours de l'infection. Des glossines naïves nourries sur des souris ne présentant des parasites que dans la peau et pas dans le sang peuvent ingérer ces parasites qui sont alors capables d'accomplir leur développement cyclique.

Pour vérifier et quantifier la présence de parasites cutanés chez l'homme, nous avons récemment mené une étude prospective dans une région endémique en Guinée où des trypanosomes ont été détectés dans les biopsies cutanées de tous les cas confirmés. De plus, $100 \%$ des cas positifs en sérologie mais non confirmés en parasitologie, et donc habituellement non traités, présentaient également des parasites au niveau du derme réticulaire. Ces observations suggèrent que les parasites cutanés sont susceptibles de contribuer au maintien de la transmission des trypanosomes. Ce réservoir cutané représente donc une nouvelle cible pour le diagnostic qui permettrait d'améliorer la sensibilité du dépistage dans le contexte de l'élimination de la THA. Différentes nouvelles pistes de diagnostic sont d'ores et déjà en cours de développement.

\section{Les affections fongiques sous-cutanées appartiennent aux « maladies tropicales négligées »}

\section{J. Chandenier}

Université de Tours, 37000 Tours, France ;

Société de pathologie exotique

jacques.chandenier@univ-tours.fr
Situées cliniquement et histologiquement en position intermédiaire entre les dermatophytoses et les mycoses invasives, les mycoses cutanées profondes (mycétomes, chromoblastomycoses, sporotrichoses, etc.) occupent une place à part en zones intertropicales.

Il s'agit d'affections sévissant surtout en zones rurales, d'origines mal connues, d'évolution chronique, et responsables de lésions invalidantes et discriminantes. Le diagnostic biologique de certitude en est souvent délicat, et les traitements, pourtant efficaces dans de nombreux cas, sont chers et peu disponibles.

Toutes ces caractéristiques ont conduit l'intégration, en 2017, de toutes ces affections fongiques dans le groupe, restreint, des pathologies identifiées en 2005 par l'OMS comme étant des «maladies tropicales négligées » (MTN). Cette classification particulière faisait suite aux souhaits de nombreux membres et partenaires de l'OMS de voir individualisées au sein d'un même groupe des pathologies transmissibles oubliées dans les grandes actions mises en place à la fin $\mathrm{du} \mathrm{XX}^{\mathrm{e}}$ siècle contre le paludisme, le VIH et la tuberculose.

Plus qu'une simple classification nosologique, ce rattachement permet de faire figurer ces pathologies dans les initiatives de prise en charge internationales, mais également locales dans les zones d'endémie où elles sévissent.

En effet, plus encore peut-être que pour tout autre type d'affections, il semble important que les acteurs de santé et les groupes de travail, au Nord comme au Sud, mutualisent les moyens disponibles et les actions entreprises dans la lutte contre ces pathologies particulières.

Dans cette lutte contre les MTN, la francophonie s'est à son tour mobilisée, via l'Organisation internationale de la francophonie, mais également, en France, au sein du réseau francophone de recherche sur les MTN d'Aviesan, et au sein de la Société de pathologie exotique qui en a fait dorénavant un axe majeur de ses actions. 